Quim. Nova, Vol. 29, No. 1, 54-60, 2006

\title{
ESTIMATIVA DO FLUXO DE AMÔNIA NA INTERFACE AR-MAR NA BAÍA DE GUANABARA - ESTUDO PRELIMINAR
}

\author{
Giselle Parno Guimarães e William Zamboni de Mello* \\ Departamento de Geoquímica, Instituto de Química, Universidade Federal Fluminense, Outeiro de São João Batista, s/n, \\ 24020-007 Niterói - RJ
}

Recebido em 24/11/04; aceito em 20/4/05; publicado na web em 24/8/05

\begin{abstract}
ESTIMATE OF AMMONIA FLUXES AT THE AIR-SEA INTERFACE IN THE GUANABARA BAY - A PRELIMINARY STUDY. In the begining of April 2004, concentrations of $\mathrm{NH}_{x}\left(\mathrm{NH}_{3}+\mathrm{NH}_{4}^{+}\right)$were measured in surface waters of the Guanabara Bay. Concentrations varied from 2 to $143 \mu \mathrm{mol} \mathrm{L}^{-1}$. Ammonia exchange at the air-sea interface was quantified using a numerical model. No measurement of $\mathrm{NH}_{3}$ concentration in air $\left(c_{\text {air }}\right)$ was performed. Thus, calculations of $\mathrm{NH}_{3}$ flux were based on the assumptions of $c_{\text {air }}=1$ and $5 \mu \mathrm{g} \mathrm{m}^{-3}$. Fluxes were predominantly from the water to the atmosphere and varied from -20 to almost $3500 \mu \mathrm{g} \mathrm{N} \mathrm{m}^{-2} \mathrm{~h}^{-1}$.
\end{abstract}

Keywords: ammonia; air-sea exchange; Guanabara Bay.

\section{INTRODUÇÃO}

$\mathrm{Na}$ atmosfera, a amônia $\left(\mathrm{NH}_{3}\right)$ é a mais importante substância gasosa com propriedade alcalina. Neste compartimento, a $\mathrm{NH}_{3}$ reage preferencialmente com os ácidos sulfúrico $\left(\mathrm{H}_{2} \mathrm{SO}_{4}\right)$ e nítrico $\left(\mathrm{HNO}_{3}\right)$, dando origem a partículas finas de sais de amônio $\left[\mathrm{NH}_{4} \mathrm{HSO}_{4},\left(\mathrm{NH}_{4}\right)_{2} \mathrm{SO}_{4}\right.$ e $\left.\mathrm{NH}_{4} \mathrm{NO}_{3}\right]$. Uma pequena parcela do $\mathrm{NH}_{3}$ $(\sim 1 \%)$ reage com o radical livre hidroxila $(\mathrm{OH})$, formando $\mathrm{NH}_{2} \mathrm{e}$ $\mathrm{H}_{2} \mathrm{O}^{1}$. Atualmente, somente cerca de $20 \%$ das emissões globais de $\mathrm{NH}_{3}$ provém de fontes naturais (e.g., solos e oceanos), o restante $(\sim 80 \%)$ tem como origem as atividades agrícolas e pecuárias, a queima de biomassa, a queima de combustíveis fosseis, a excreta humana e os catalisadores de veículos ${ }^{1-5}$.

Vários estudos realizados em grandes centros urbanos e industriais no Brasil mostram que, em unidade molar, o aporte atmosférico (via deposição úmida) de $\mathrm{NH}_{4}^{+}$é tão alto quanto ou, na maioria das vezes, superior ao de nitrato $\left(\mathrm{NO}_{3}^{-}\right)^{6-11}$. A elevada deposição atmosférica de $\mathrm{NH}_{4}^{+}$pode contribuir para acidificação de ambientes terrestres e aquáticos (a nitrificação resulta na formação de dois hidrogênios ácidos: $\mathrm{NH}_{4}{ }^{+}+2 \mathrm{O}_{2} \rightarrow 2 \mathrm{H}^{+}+\mathrm{NO}_{3}{ }^{-}+\mathrm{H}_{2} \mathrm{O}$ ), eutrofização de ecossistemas aquáticos e aumento da produção e emissão de óxido nitroso $\left(\mathrm{N}_{2} \mathrm{O}\right)^{2,12,13}$.

Nos ambientes aquáticos naturais, o nitrogênio $(\mathrm{N})$ amoniacal (representado por $\mathrm{NH}_{\mathrm{x}}$ ) compreende as formas do íon amônio $\left[\mathrm{NH}_{4}^{+}\right] \mathrm{e}$ amônia $\left[\mathrm{NH}_{3}\right]$ dissolvidos, e a proporção entre elas depende de temperatura, salinidade e, principalmente, $\mathrm{pH}^{14,15}$. Nos oceanos, o $\mathrm{NH}_{\mathrm{x}}$ origina-se da decomposição (aeróbica e anaeróbica) de substâncias orgânicas e excreção de metazoários. Na água do mar, os principais processos consumidores do $\mathrm{NH}_{x}$ são a oxidação microbiológica (via bactérias nitrificadoras), a assimilação pelo fitoplâncton e bactérias, e a transferência para a atmosfera como $\mathrm{NH}_{3}{ }^{16}$. Entretanto, a direção do fluxo do gás na interface ar-mar (i.e., da atmosfera para o mar ou do mar para a atmosfera) depende das concentrações nos dois compartimentos (atmosfera e mar) ${ }^{17-19}$. Diferentes regiões marinhas podem exercer o papel de fonte ou sorvedouro de $\mathrm{NH}_{3}{ }^{2}$. O conhecimento das fontes, naturais ou antrópicas, bem como de suas grandezas, é necessário para um melhor entendimento do ciclo do $\mathrm{N}$ e avaliação dos impactos causados pelas atividades humanas aos ambientes naturais.

*e-mail: zamboni@geoq.uff.br
$\mathrm{Na}$ água do mar, as concentrações de $\mathrm{NH}_{\mathrm{x}}$ encontram-se na faixa de nanomolar $\left(1 \mathrm{nmol} \mathrm{L}^{-1}=10^{-9} \mathrm{~mol} \mathrm{~L}^{-1}\right)$, normalmente menores que $100 \mathrm{nmol} \mathrm{L}^{-1}{ }^{16}$. As concentrações mais elevadas ocorrem após as florações de fitoplâncton e nas regiões costeiras, onde as concentrações encontram-se normalmente na faixa de 100 a $1000 \mathrm{nmol} \mathrm{L}^{-1}$, mas podendo atingir até $5000 \mathrm{nmol} \mathrm{L}^{-1}$ $\left.(5 \mu \mathrm{mol} \mathrm{L})^{-1}\right)$.

Nas águas da Baía de Guanabara, alguns estudos mostram concentrações de $\mathrm{NH}_{x}$ que atingem níveis bastante superiores àqueles verificados nas regiões oceânicas. A Baía de Guanabara é caracterizada como um sistema costeiro poluído e eutrófico, onde as principais fontes de poluição são os esgotos domésticos ${ }^{20}$. Vários trabalhos foram realizados em suas águas, com acompanhamento de vários parâmetros físico-químicos, inclusive $\mathrm{NH}_{x}{ }^{21-29}$. A maior parte destes estudos utilizou dados de monitoramento da Fundação Estadual de Meio Ambiente do estado do Rio de Janeiro (FEEMA). Os trabalhos de Lavrado et al. ${ }^{22}$ e Paranhos e Mayr ${ }^{23}$ compilaram dados do período 1980-1990, e Kjerfve et al. ${ }^{26}$ de 1980 a 1993. Todos estes estudos mostram grande variação espacial nas concentrações de $\mathrm{NH}_{x}$, sendo o setor noroeste da baía aquele cujas concentrações são mais altas, em virtude da elevada descarga de esgotos domésticos e da pouca renovação das águas, devido à pequena profundidade.

$\mathrm{Na}$ atmosfera oceânica, de acordo com os dados compilados por Duce et al..$^{18}$, as concentrações de $\mathrm{NH}_{3}$ encontram-se em torno $0,05 \mu \mathrm{g} \mathrm{m}^{-3}\left(1,0 \mu \mathrm{g} \mathrm{NH}_{3} \mathrm{~m}^{-3}=1,44 \mathrm{ppb}\right.$, para $T=25{ }^{\circ} \mathrm{C}$ e $P=1,0$ atm), com variações de 0,01 a $0,6 \mu \mathrm{g} \mathrm{m}^{-3}$. No Brasil, a literatura mostra que, de modo geral, as concentrações de $\mathrm{NH}_{3}$ na atmosfera apresentam valores médios inferiores a $1 \mu \mathrm{g} \mathrm{m}^{-3}$ em áreas não poluídas, de 1 a $5 \mu \mathrm{g} \mathrm{m}^{-3}$ em áreas urbanas e de pastagens, e superiores a $5 \mu \mathrm{g} \mathrm{m}^{-3} \mathrm{em}$ áreas influenciadas pelas atividades industriais. Por ex., no Recôncavo Baiano, Campos ${ }^{30}$ encontrou concentrações médias de $\mathrm{NH}_{3}$ no ar que variaram de $<0,1$ a $1,0 \mu \mathrm{g} \mathrm{m}^{-3}$ nas áreas distantes de fontes de poluição e de 4,2 a $6,6 \mu \mathrm{g} \mathrm{m}^{-3}$ em áreas influenciadas por emissões industriais. Na Amazônia, em áreas dominadas por pastagens, as concentrações médias de $\mathrm{NH}_{3}$ foram de $0,9 \mu \mathrm{g} \mathrm{m}^{-3}$ no período chuvoso e $4,5 \mu \mathrm{g} \mathrm{m}^{-3}$ no período seco, enquanto que na floresta (período seco) a média foi de $2,0 \mu \mathrm{g} \mathrm{m}^{-3} 31$. Em São Paulo, Fornaro et al. ${ }^{32}$ mediram concentrações de $\mathrm{NH}_{3}$ durante o inverno, obtendo média de $1,3 \mu \mathrm{g} \mathrm{m}^{-3}$ na Cidade Univer- 
sitária e 1,6 $\mu \mathrm{g} \mathrm{m}^{-3}$ na Água Funda; a concentração máxima verificada foi de aproximadamente $4,2 \mu \mathrm{g} \mathrm{m}^{-3}$. Na cidade de Araraquara (SP), concentrações de $\mathrm{NH}_{3}$ no ar variaram de 0,3 a 4,2 $\mu \mathrm{g} \mathrm{m}^{-3} 5$. Em estudo realizado em nove municípios da Baixada Fluminense (a maioria no setor norte da região metropolitana do Rio de Janeiro), Maia ${ }^{33}$ verificou, em setembro de 2000, concentrações de $\mathrm{NH}_{3}$ de 2,2 a 91,4 $\mu \mathrm{g} \mathrm{m}^{-3}$, com média aritmética e mediana de 12,9 e $7,2 \mu \mathrm{g} \mathrm{m}^{-3}$, respectivamente.

O presente trabalho tem como objetivo estimar o fluxo de $\mathrm{NH}_{3}$ na interface ar-mar na Baía de Guanabara. Para isso, utilizou-se o modelo descrito por Asman et al. ${ }^{19}$ e Quinn et $a l .{ }^{16}$, ao qual foram aplicados valores de $\mathrm{NH}_{x}, \mathrm{pH}$, salinidade e temperatura da água de medidas realizadas no período de 5 a 7/4/2004. Não foram realizadas medidas de $\mathrm{NH}_{3}$ na atmosfera; sendo assim, adotaram-se as concentrações de 1,0 e $5,0 \mu \mathrm{g} \mathrm{m}^{-3}$ para as condições de ventos de origem marinha e continental, respectivamente.

\section{PARTE EXPERIMENTAL}

\section{Área de estudo}

Dos $850 \mathrm{~km}$ de extensão litorânea do estado do Rio de Janeiro, $131 \mathrm{~km}$ correspondem ao perímetro da Baía de Guanabara, que possui $384 \mathrm{~km}^{2}$ de área superficial total, $328 \mathrm{~km}^{2}$ de espelho d'água, $1,9 \times 10^{9} \mathrm{~m}^{3}$ de volume d'água médio, $4.080 \mathrm{~km}^{2}$ de bacia de drenagem e $100 \pm 60 \mathrm{~m}^{3} \mathrm{~s}^{-1}$ de vazão hídrica, que é principalmente atribuída (ca. $75 \%$ ) aos 35 rios que drenam em direção à baía e o restante (ca. 25\%) à transposição de águas do rio Paraíba do $\mathrm{Sul}^{21,26,34}$. A profundidade da baía não é uniforme. Ao longo do canal principal, que segue o eixo principal da baía, a profundidade média é de $20 \mathrm{~m}$, enquanto que no extremo noroeste da baía a profundidade é em torno de $1 \mathrm{~m}$ durante a baixa-mar ${ }^{21}$.

Dos 11 milhões de habitantes residentes na região metropolitana do Rio de Janeiro, cerca de 7,8 milhões geram esgotos domésticos que são lançados diretamente na baía ${ }^{25}$.

A bacia da Baía de Guanabara possui o segundo maior parque industrial do país, com cerca de 6000 indústrias, das quais cerca de $1 \%$ é responsável por $80 \%$ da poluição industrial lançada às águas da baía ${ }^{20}$. A baía possui 16 terminais marítimos de petróleo, 2 portos comerciais, 2 refinarias de petróleo, 13 estaleiros e recebe $18,7 \mathrm{~m}^{3} \mathrm{~s}^{-1}$ de esgotos domésticos ${ }^{34}$.

\section{Coleta de amostras}

Para este estudo, foram coletadas amostras de água em 37 estações de coleta, previamente definidas com base numa malha que contemplava um total de 44 estações eqüidistantes $(2 \mathrm{~km})$ entre si. Entretanto, a logística impediu o acesso às estações situadas em alguns locais mais rasos da baía, principalmente os situados na área de Proteção Ambiental de Guapimirim, no segmento nordeste da baía. A campanha de coleta foi realizada nos dias 5, 6 e 7/4/2004, utilizando-se a embarcação "Mar de Thetys" da PróReitoria de Extensão da UFF. A seqüência de coleta nas 37 estações e os dias em que foram realizadas encontram-se indicados na Figura 1.

As amostras foram coletadas, sempre que possível no período de maré enchente, com garrafa de van Dorn com volume de 5 $\mathrm{L}$, a uma profundidade entre 0,5 e 1,0 $\mathrm{m}$. A tábua de maré prevista foi obtida junto à Marinha do Brasil, na página da Diretoria de Hidrografia e Navegação (DHN) ${ }^{35}$. Nas estações de coleta foram medidos diretamente na água os parâmetros salinidade, $\mathrm{pH}$ e temperatura com equipamentos Handylab LF1/LF 613T e WTW

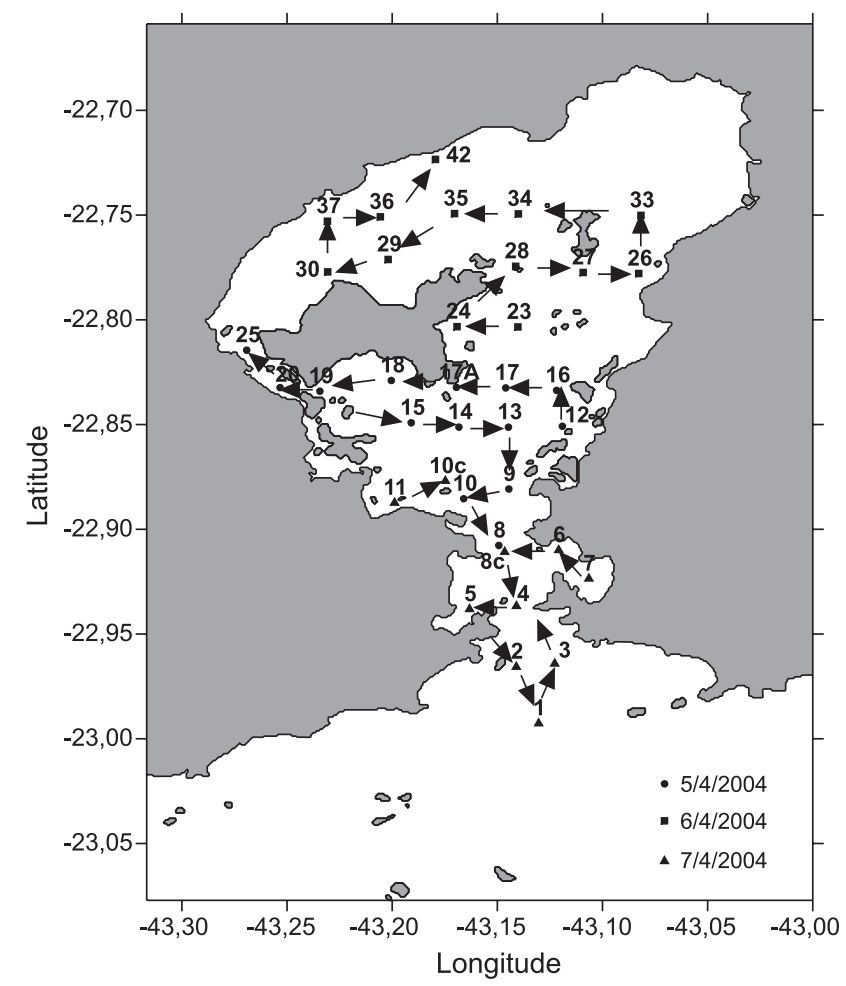

Figura 1. Distribuição das estações de amostragem na Baía de Guanabara, com representação das trajetórias por data de amostragem, período de 5-7 de abril de 2004. A campanha de amostragem teve início na estação de número 7 e fim na estação de número 3

pH330/SET-1. As amostras foram acondicionadas em dois frascos de polipropileno de 250 e $500 \mathrm{~mL}$, mantidos na ausência de luz e sob refrigeração (em gelo) até a chegada ao laboratório. Também a bordo, 3 alíquotas de $5 \mathrm{~mL}$ de amostra de água foram transferidas para frascos de vidro âmbar de $60 \mathrm{~mL}$ (com tampa rosqueada), para quantificação do nitrogênio amoniacal $\left(\mathrm{NH}_{\mathrm{x}}\right)$.

Os valores de velocidade do vento, correspondentes ao momento de cada coleta de amostra, foram obtidos dos arquivos de dados das estações meteorológicas do campus do Gragoatá $\left(22^{\circ}\right.$ 53' 49" S, 43 07' 51" W; Universidade Federal Fluminense) e da Ilha do Fundão (Universidade Federal do Rio de Janeiro). Para os dias 5 e 6/4/2004 foram utilizadas as velocidades médias horárias dos ventos da estação da Ilha do Fundão-UFRJ, enquanto que para o dia 7/4/2004 foram utilizadas as velocidades médias horárias dos ventos da estação do campus Gragoatá-UFF.

\section{Determinação de nitrogênio amoniacal na água do mar}

$\mathrm{O}$ método aplicado para determinação do $\mathrm{NH}_{\mathrm{x}}$ foi o do azul de indofenol ${ }^{36}$. O método consiste na reação do $\mathrm{NH}_{\mathrm{x}}\left(\mathrm{NH}_{3}+\mathrm{NH}_{4}^{+}\right)$com cloro para formação da monocloramina, que reage com o fenol, formando um complexo de coloração azul ${ }^{15}$. Para isso foram adicionados 0,2 $\mathrm{mL}$ de solução de fenol, $0,2 \mathrm{~mL}$ de ácido dicloroisocianúrico (Trione) e $0,2 \mathrm{~mL}$ de citrato de sódio a $5 \mathrm{~mL}$ de amostra em frascos de vidro âmbar de $60 \mathrm{~mL}$. A adição dos reagentes às amostras foi efetuada a bordo, imediatamente após a coleta, para evitar contaminação das amostras. No mesmo dia da coleta, em laboratório, foram realizadas as medidas de absorbância de padrões e amostras em comprimento de onda de $630 \mathrm{~nm}$ em um espectrofotômetro Hitachi modelo U-1100. O limite de detecção das análises de $\mathrm{NH}_{\mathrm{x}}$ foi de 1,0 $\mu \mathrm{mol} \mathrm{L} \mathrm{L}^{-1}$ e a reprodutibilidade dos resultados para análises realizadas em triplicata encontrava-se entre $\pm 10 \%$. 


\section{Cálculo do fluxo de $\mathrm{NH}_{3}$ na interface ar-mar}

O fluxo $(F)$ de um gás na interface ar-mar pode ser calculado a partir de um modelo baseado na diferença entre as concentrações do gás na fase líquida e na fase gasosa $(\Delta \mathrm{C}$ - termo que define a direção do fluxo) e um parâmetro cinético ( $k$ - termo que define a intensidade do fluxo) $)^{17}$

$F=k \Delta \mathrm{C}$

A variável $k$ representa a velocidade de transferência ("transfer velocity") ou velocidade de troca ("exchange velocity"). O termo $\Delta \mathrm{C}$ é obtido a partir de medidas diretas do gás na superfície do mar e na atmosfera adjacente.

No caso da amônia $\left(\mathrm{NH}_{3}\right)$, da mesma forma que o dióxido de enxofre $\left(\mathrm{SO}_{2}\right)$ e o vapor d'água, a velocidade de transferência do gás na interface ar-mar é controlada predominantemente pela resistência ao transporte em fase gasosa $\left(r_{\mathrm{a}}\right)$. Neste caso, a velocidade de transferência em fase gasosa $\left(k_{\mathrm{a}}\right)$, expressa em $\mathrm{m} \mathrm{s}^{-1}$, é a recíproca da resistência e pode ser calculada a partir da Equação abaixo, adotada para gases solúveis em água ${ }^{18}$

$k_{\mathrm{a}}=1 / r_{\mathrm{a}}=u /\left[770+45(M)^{1 / 3}\right]$

onde $u$ representa a velocidade do vento $\left(\mathrm{m} \mathrm{s}^{-1}\right)$ e $M$ é a massa molecular do gás.

O fluxo de $\mathrm{NH}_{3}\left(\mu \mathrm{g} \mathrm{m}^{-2} \mathrm{~s}^{-1}\right)$ na interface ar-mar é calculado a partir da seguinte Equação

$F=k_{\mathrm{a}}\left(c_{\mathrm{eq}}-c_{\mathrm{ar}}\right)$

na qual $c_{\text {eq }}$ é a concentração teórica de $\mathrm{NH}_{3}$ no $\operatorname{ar}\left(\mu \mathrm{g} \mathrm{NH}_{3} \mathrm{~m}^{-3}\right)$ em equilíbrio com a concentração medida de $\mathrm{NH}_{\mathrm{x}}\left(\mathrm{NH}_{4}^{+}+\mathrm{NH}_{3}\right)$ na superfície do mar e $c_{\text {ar }}$ é a concentração medida de $\mathrm{NH}_{3}$ no ar $(\mu \mathrm{g}$ $\mathrm{NH}_{3} \mathrm{~m}^{-3}$ ). $\mathrm{O}$ valor de $F$ possui sinal positivo sempre que a transferência se der do mar para a atmosfera (i.e., o mar atua como uma fonte de $\mathrm{NH}_{3}$ para a atmosfera) e negativo sempre que a transferência se der da atmosfera para o mar (i.e., a atmosfera atua como uma fonte de $\mathrm{NH}_{3}$ para o mar).

Para se calcular $c_{\text {eq }}$ utiliza-se a seguinte expressão ${ }^{16,19}$

$c_{\mathrm{eq}}=M\left[\mathrm{NH}_{\mathrm{x}}\right]_{\mathrm{mar}} / R T H_{\mathrm{NH} 3}\left\{\left[1 / \gamma_{\mathrm{NH} 3}\right]+\left[10^{-\mathrm{pH}} /\left(\gamma_{\mathrm{NH} 4} K_{\mathrm{NH} 4}\right)\right]\right\}$

onde $M$ é a massa molecular da amônia $\left(17,03 \mathrm{~g} \mathrm{~mol}^{-1}\right),\left[\mathrm{NH}_{\mathrm{x}}\right]_{\operatorname{mar}}$ é a concentração de $\mathrm{NH}_{x}$ na água do mar $\left(\mu \mathrm{mol} \mathrm{L}^{-1}\right), R$ é a constante dos gases perfeitos $\left(8,2075 \times 10^{-5} \mathrm{~atm} \mathrm{~m}^{3} \mathrm{~mol}^{-1} \mathrm{~K}^{-1}\right), T$ é a temperatura $(K), H_{\mathrm{NH} 3}$ é a constante da Lei de Henry $\left(\mathrm{mol} \mathrm{L}^{-1} \mathrm{~atm}^{-1}\right), \mathrm{pH}$ representa o $\mathrm{pH}$ medido da água do mar, $\gamma_{\mathrm{NH} 3}$ é o coeficiente de atividade da $\mathrm{NH}_{3}$ na água do mar, $\gamma_{\mathrm{NH} 4}$ é o coeficiente de atividade do íon amônio $\left(\mathrm{NH}_{4}^{+}\right)$na água do mar e $K_{\mathrm{NH}_{4}}$ é a constante de dissociação do $\mathrm{NH}_{4}^{+}\left(\mu \mathrm{mol} \mathrm{L}{ }^{-1}\right)$.

A constante da Lei de Henry para $\mathrm{NH}_{3}$ é calculada em função da temperatura $(T)$ a partir da Equação ${ }^{16,19,37}$

$H=56 \exp [4092(1 / T-1 / 298,15)]$

$\mathrm{O}$ coeficiente de atividade da $\mathrm{NH}_{3}\left(\gamma_{\mathrm{NH} 3}\right)$ em função da força iônica $(I)$ foi calculado por Randall e Failey ${ }^{38}$ em solução de $\mathrm{NaCl}$ a $25{ }^{\circ} \mathrm{C}$ e pode ser obtido a partir da seguinte Equação ${ }^{16,19,39}$

$\gamma_{\mathrm{NH} 3}=1+0,085 I$

onde $I$ corresponde à força iônica da água do mar calculada a partir da salinidade $(S)$, através da Equação $0^{16,19,40}$

$I=0,00147+0,01988 S+2,08357 \times 10^{-5} S^{2}$

O coeficiente de atividade de um íon $i$ pode ser calculado a partir da Equação de Davies ${ }^{14,16}$

$\log \gamma_{i}=-\mathrm{A} z_{i}^{2}\left\{\left[I^{1 / 2} /\left(1+I^{1 / 2}\right)\right]-0,2 I\right\}$

onde A é calculado a partir da equação polinomial $\mathrm{A}=2,719 \times 10^{-6}$ $T^{2}-7,378 \times 10^{-4} T+0,4883^{39,41}$ e $z_{i}$ corresponde à carga do íon $i$. Neste caso, $i$ representa o íon $\mathrm{NH}_{4}^{+} \mathrm{e}$, sendo assim, $\gamma_{i}=\gamma_{\mathrm{NH} 4}$.

A constante de dissociação do $\mathrm{NH}_{4}^{+}\left(K_{\mathrm{NH} 4}\right)$ varia com a temperatura e é calculada a partir da Equação ${ }^{16,19,42}$

$K_{\mathrm{NH} 4}=5,67 \times 10^{-10} \exp [-6286(1 / T-1 / 298,15)]$

\section{RESULTADOS E DISCUSSÃO}

\section{Nitrogênio amoniacal e demais parâmetros físico-químicos nas águas da Baía de Guanabara}

A estatística descritiva das concentrações de nitrogênio amoniacal $\left(\mathrm{NH}_{\mathrm{x}}\right)$, temperatura, salinidade e $\mathrm{pH}$, medidos entre os dias 5 e 7/4/ 2004 em amostras de águas superficiais da Baía de Guanabara, encontra-se compilada na Tabela 1 . Do total de amostras $(n=37), 23$ foram coletadas durante a maré enchente, 3 na vazante, 6 na preamar e 5 na baixa-mar. As concentrações de $\mathrm{NH}_{x}$ variaram de 2,3 a $143 \mu \mathrm{mol} \mathrm{L}{ }^{-1}$. A média aritmética $\left(26,2 \mu \mathrm{mol} \mathrm{L}^{-1}\right)$ é cerca de 3 vezes superior à mediana $\left(8,7 \mu \mathrm{mol} \mathrm{L}^{-1}\right)$, evidenciando a forte influência de um pequeno número de amostras cujas concentrações foram bem maiores em relação às demais. Vale salientar que estas medidas de tendência central não devem representar os níveis de $\mathrm{NH}_{\mathrm{x}}$ nas águas superficiais da baía ao longo do ano. Em estudo realizado na década de 80, Mayr et al. ${ }^{21}$ mostraram que a média de concentração de $\mathrm{NH}_{\mathrm{x}}$ no período de verão foi menor que a de inverno. Essa variação anual pode ser explicada em função da maior diluição causada pelas chuvas e maior assimilação de nutrientes pela produtividade primária durante o verão.

Tabela 1. Estatística descritiva das concentrações de nitrogênio amoniacal $\left(\mathrm{NH}_{\mathrm{x}}\right)$, temperatura $(\mathrm{T})$, salinidade $(\mathrm{S})$ e $\mathrm{pH}$ nas águas superficiais de 37 estações de amostragem na Baía de Guanabara, no período de 5 a 7 de abril de 2004

\begin{tabular}{lcrrr}
\hline & $\mathrm{NH}_{\mathrm{x}}\left(\mu \mathrm{mol} \mathrm{L} \mathrm{L}^{-1}\right)$ & $T\left({ }^{\circ} \mathrm{C}\right)$ & \multicolumn{1}{c}{$S$} & $\mathrm{pH}$ \\
\hline Média aritmética & 26,2 & 27,6 & 30,8 & 8,52 \\
Desvio padrão & 38,7 & 1,1 & 2,9 & 0,18 \\
Mediana & 8,7 & 27,8 & 30,0 & 8,50 \\
Mínimo & 2,3 & 25,0 & 25,8 & 8,02 \\
Máximo & 143,2 & 29,6 & 35,2 & 8,85 \\
\hline
\end{tabular}

* Média aritmética calculada a partir das concentrações de $\mathrm{H}^{+}(\mathrm{pH}$ $\left.=-\log \left[\mathrm{H}^{+}\right]\right)$.

Concentrações de $\mathrm{NH}_{\mathrm{x}}$ superiores a $80 \mu \mathrm{mol} \mathrm{L} \mathrm{L}^{-1}$ foram encontradas nas estações 10, 18, 20, 25 e 30 (Figura 2). As estações 20 $\left.(101 \mu \mathrm{mol} \mathrm{L})^{-1}\right)$ e $25\left(143 \mu \mathrm{mol} \mathrm{L}{ }^{-1}\right)$ estão localizadas entre a costa oeste da Ilha do Governador e o continente. Os níveis elevados de $\mathrm{NH}_{\mathrm{x}}$ nestas estações, principalmente na 25 , podem ser atribuídos à carga de esgotos domésticos associada aos cursos d'água que deságuam neste trecho da baía, tais como os rios São João de Meriti e Irajá e canal da Penha, face à proximidade de suas desembocadu- 


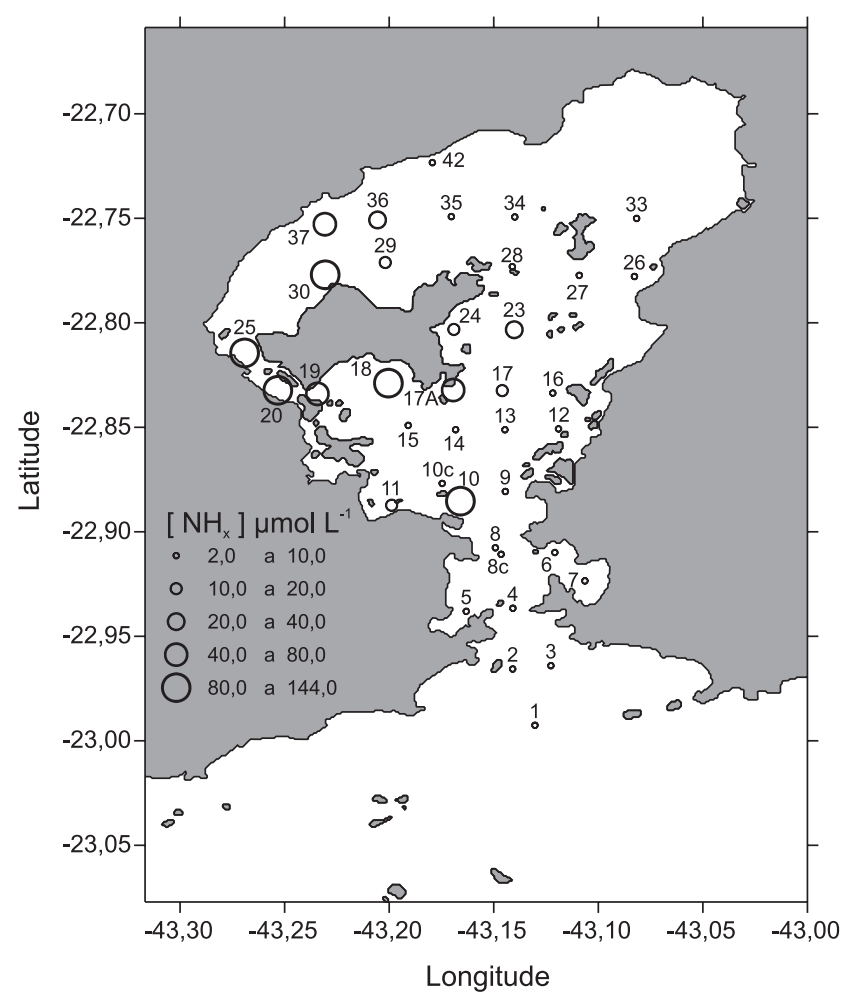

Figura 2. Faixas de concentração de nitrogênio amoniacal (em $\mu \mathrm{mol} \mathrm{L}^{-1}$ ) em águas superficiais da Baía de Guanabara, obtidas com base em resultados de 37 medições realizadas no período de 5-7 de abril de 2004. Os números na figura representam as estações de amostragem

ras. A descarga de poluição dos rios juntamente com a profundidade baixa e pouca circulação de água neste trecho da baía de Guanabara são os fatores controladores dos elevados níveis de $\mathrm{NH}_{\mathrm{x}}$ verificados, já relatados em outros estudos ${ }^{22,43}$. A estação $30(88$ $\mu \mathrm{mol} \mathrm{L} \mathrm{L}^{-1}$ ) localiza-se a cerca de $1 \mathrm{~km}$ a noroeste da Ponta de Tubiacanga, na costa norte da Ilha do Governador, e a estação 18 $\left(85 \mu \mathrm{mol} \mathrm{L}{ }^{-1}\right)$, a aproximadamente $1,5 \mathrm{~km}$ ao sul da Ponta de Santa Cruz, na costa sul da Ilha do Governador. A estação 10 (142 $\mu \mathrm{mol}$ $\left.\mathrm{L}^{-1}\right)$, localizada a cerca de $1 \mathrm{~km}$ a sudeste da Ilha das Enxadas, foi a única estação nas proximidades do canal central da baía que apresentou concentração de $\mathrm{NH}_{x}$ tão elevada. Considerando que a coleta neste ponto ocorreu durante a maré vazante, este fato pode estar associado à descarga do canal do Mangue e/ou à uma contribuição oriunda dos setores oeste e sul da Ilha do Governador. Concentrações entre 40 e $80 \mu \mathrm{mol} \mathrm{L}{ }^{-1}$ foram verificadas nas estações 17A, 19 e 37. As estações 17A $\left(68 \mu \mathrm{mol} \mathrm{L}^{-1}\right)$ e $19\left(65 \mu \mathrm{mol} \mathrm{L}^{-1}\right)$ estão localizadas ao sul da Ilha do Governador, enquanto a estação 37 (43 $\mu \mathrm{mol} \mathrm{L} \mathrm{L}^{-1}$ ) se localiza ao norte da Ilha, nas proximidades da foz do rio Iguaçu.

Em abril de 2004, as estações que apresentaram as concentrações mais altas foram aquelas situadas a sudoeste, oeste e noroeste da Ilha do Governador. Lavrado et al..$^{22}$ mostraram, para 6 estações de amostragem monitoradas no período de 1980 a 1990 , concentrações médias de $\mathrm{NH}_{\mathrm{x}}$ entre 4,1 a $199 \mu \mathrm{mol} \mathrm{L}^{-1}$ e estimaram que o crescimento das concentrações de $\mathrm{NH}_{x}$ na década de 80 variou de 9 a $28 \mu \mathrm{mol} \mathrm{L}^{-1}$ ano $^{-1}$. Medidas de $\mathrm{NH}_{\mathrm{x}}$ realizadas em águas superficiais da Baía de Guanabara em agosto de 1990 mostram concentrações de até $650 \mu \mathrm{mol} \mathrm{L}{ }^{-1}$ no trecho entre a Ilha do Governador e a Ilha do Fundão ${ }^{28}$.

Com base em resultados de três campanhas realizadas na Baía de Guanabara, em maio, junho e outubro de 1992, Valentin et al. ${ }^{29}$ encontram uma concentração média de $\mathrm{NH}_{\mathrm{x}}$ para as águas superfi- ciais de $16,5 \mu \mathrm{mol} \mathrm{L} \mathrm{L}^{-1}$ e verificaram, comparando resultados das mesmas estações de amostragem, concentrações de $\mathrm{NH}_{\mathrm{x}}$ mais altas durante a maré enchente que na vazante. Em abril de 2004, verificaram-se concentrações mais baixas durante a maré enchente $(29,2$ $\left.\mu \mathrm{mol} \mathrm{L} \mathrm{L}^{-1} ; \mathrm{n}=23\right)$ que na vazante $\left(52,9 \mu \mathrm{mol} \mathrm{L}^{-1} ; \mathrm{n}=3\right)$. Por outro lado, verificou-se que a concentração média das amostras coletadas durante a preamar $\left(16,6 \mu \mathrm{mol} \mathrm{L} \mathrm{L}^{-1} ; \mathrm{n}=6\right)$, momento em que a maré atinge seu nível máximo, foi superior à concentração média das amostras coletadas durante a baixa-mar $\left(8,3 \mu \mathrm{mol} \mathrm{L}^{-1} ; \mathrm{n}=5\right)$, momento em que a maré atinge seu nível mínimo. Entretanto, no caso da campanha de abril de 2004, as variações verificadas podem estar relacionadas à localização das estações, por isso as comparações entre conjuntos de estações distintas limitam-se a interpretações relativas entre condições de maré e concentrações de $\mathrm{NH}_{x}$.

As médias aritméticas e desvios padrões da temperatura e salinidade para o período 5-7/4/2004 foram respectivamente 27,6 \pm $1,1{ }^{\circ} \mathrm{C}$ e $30,8 \pm 2,9$. Paranhos e Mayr ${ }^{23}$ compilaram as médias mensais de temperatura e salinidade, em águas superficiais da Baía de Guanabara, correspondentes ao período 1980-90, mostrando que as médias de temperatura variaram de aproximadamente $22{ }^{\circ} \mathrm{C}$ (em julho) a $28{ }^{\circ} \mathrm{C}$ (em janeiro e fevereiro) e as médias de salinidade, de aproximadamente 21 (em fevereiro) a 29 (em outubro). A média de temperatura verificada no início de abril de 2004 é compatível com as médias apresentadas por outros estudos para este período do ano, i.e., que são de aproximadamente $27^{\circ} \mathrm{C}$ em março e $25^{\circ} \mathrm{C}$ em abril, enquanto as médias de salinidade apresentadas para os meses de março e abril são em torno de $27^{21,23}$, inferior à que se verificou em início de abril de 2004 (30,8). A maior salinidade média verificada em abril de 2004 em relação àquelas relatadas por outros autores para o mesmo período do ano pode ser explicada pelo fato de que $62 \%$ das amostras foram coletadas durante a maré enchente, além de que houve um maior número de amostras coletadas ao longo do canal central em relação a outros estudos, trecho onde se verifica grande influência das águas oceânicas. Verificou-se uma forte correlação negativa entre salinidade e temperatura $(r=-0,76 ; p<0,05)$ (Figura 3), tal como verificado por Mayr et al. ${ }^{21}$ e Paranhos e Mayr ${ }^{23}$, indicando a influência das águas de origem continental na temperatura das águas superficiais da Baía de Guanabara.

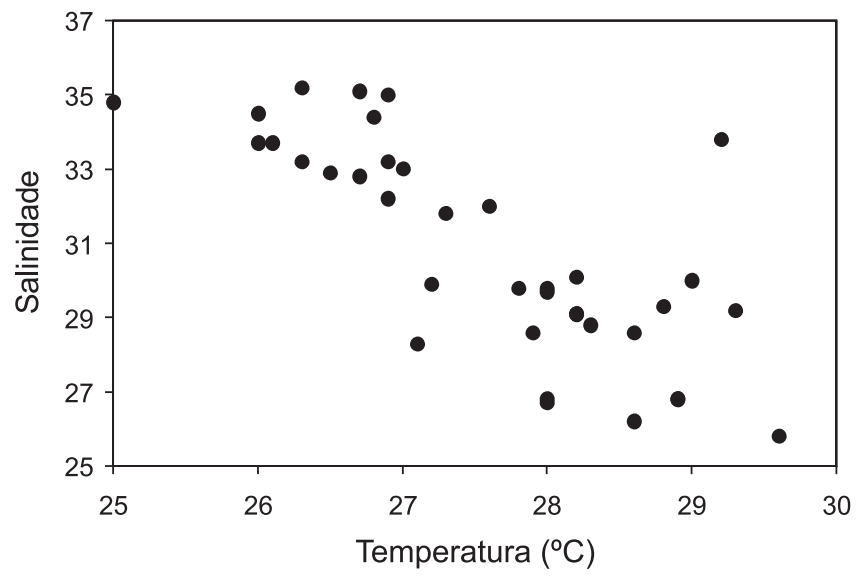

Figura 3. Relação salinidade vs. temperatura nas estações de amostragem no período 5-7 de abril de 2004

O valor médio de $\mathrm{pH}$ em abril de 2004 foi de $8,52 \pm 0,18$. Quiroga $^{44}$, que realizou coletas trimestrais durante o período chuvoso e seco em 5 pontos da baía, e Contador ${ }^{45}$, que realizou coletas quinzenais na entrada e na altura da ponte Rio-Niterói, encontraram $\mathrm{pH}$ médios de 8,45 $\pm 0,28$ e 8,13 $\pm 0,24$, respectivamente. Durante as campanhas realizadas pelo "Japan International 
Cooperation Agency"25, para o Programa de Despoluição da Baía de Guanabara, foi verificado $\mathrm{pH}$ médio de 8,47 $\pm 0,75$. Portanto, o valor de $\mathrm{pH}$ médio obtido em abril de 2004 é bastante compatível com aqueles apresentados em outros estudos. Os fluxos de $\mathrm{NH}_{3}$ na interface ar-mar são bastante sensíveis tanto às concentrações de $\mathrm{NH}_{\mathrm{x}}$ quanto ao $\mathrm{pH}$ da água do $\operatorname{mar}^{16,19,46}$.

Com base em 42 amostras de água superficial coletadas, em um único dia de agosto de 2000, nas enseadas de Icaraí e Jurujuba, Santos ${ }^{47}$ encontrou concentrações de $\mathrm{NH}_{x}$ entre 0,5 e $17,4 \mu \mathrm{mol}$ $\mathrm{L}^{-1}$, com média aritmética e desvio padrão de 5,5 $\pm 4,0 \mu \mathrm{mol} \mathrm{L} \mathrm{L}^{-1}$. Os valores médios de temperatura, salinidade e $\mathrm{pH}( \pm$ desvio padrão) apresentados por Santos ${ }^{47}$ foram $21,0{ }^{\circ} \mathrm{C}\left( \pm 0,4{ }^{\circ} \mathrm{C}\right), 33,8( \pm$ $1,4)$ e $8,6( \pm 0,1)$. Nossos resultados, verificados em abril de 2004, para as estações 6 e 7, i.e., Icaraí e Jurujuba, foram $\mathrm{NH}_{\mathrm{x}}(2,7$ e $\left.4,3 \mu \mathrm{mol} \mathrm{L} \mathrm{L}^{-1}\right)$, temperatura $\left(26,3\right.$ e $\left.26,9{ }^{\circ} \mathrm{C}\right)$, salinidade $(35,2 \mathrm{e}$ $35,0)$ e $\mathrm{pH}\left(8,08\right.$ e 8,02). Embora os resultados de $\mathrm{NH}_{\mathrm{x}}$ e demais parâmetros obtidos em abril de 2004 possam não ser representativos das enseadas citadas, as diferenças encontradas entre nossos resultados e os valores médios de $\operatorname{Santos}^{47}$ devem, pelo menos em parte, estar associadas à sazonalidade.

\section{Fluxos de amônia na interface ar-mar}

Os resultados dos cálculos dos fluxos $(F)$ de $\mathrm{NH}_{3}$ na interface ar-mar para a Baía de Guanabara são apresentados na Tabela 2. Os valores calculados das concentrações de $\mathrm{NH}_{3}$ no ar em equilíbrio $\left(c_{\mathrm{eq}}\right)$ com as concentrações medidas de $\mathrm{NH}_{\mathrm{x}}$ nas águas da baía mostram variações superiores a 2 ordens de grandeza, i.e., variaram de 1,8 a $290 \mu \mathrm{g} \mathrm{NH}_{3} \mathrm{~m}^{-3}$. A média aritmética e a mediana dos valores calculados de $c_{\text {eq }}$ foram respectivamente, 48,5 e $14,3 \mu \mathrm{g} \mathrm{NH}_{3} \mathrm{~m}^{-3}$. Apesar de não terem sido feitas medidas de concentrações de $\mathrm{NH}_{3}$ no ar durante o período de estudo, os resultados mostram que $73 \%$ dos 37 valores de $c_{\text {eq }}$ calculados foram pelo menos 10 vezes maiores que o menor valor atribuído à concentração de $\mathrm{NH}_{3}$ no ar $\left(c_{\mathrm{ar}}\right)$ (i.e., $1,0 \mu \mathrm{g} \mathrm{NH}_{3} \mathrm{~m}^{-3}$ ), adotado para simulação dos fluxos de $\mathrm{NH}_{3}$ na interface ar-mar. Dos 37 valores de $c_{\text {eq }}$ calculados, $24 \%$ foram pelo menos 10 vezes maiores que o maior valor atribuído à $c_{\mathrm{ar}}$ (i.e., $5,0 \mu \mathrm{g} \mathrm{NH}_{3} \mathrm{~m}^{-3}$ ), adotado para simulação dos fluxos de $\mathrm{NH}_{3}$. Isto sugere que, no caso da Baía de Guanabara, a concentração de $\mathrm{NH}_{\mathrm{x}}$ na água exerce maior controle no fluxo de $\mathrm{NH}_{3}$ na interface ar-mar que a concentração de $\mathrm{NH}_{3}$ no ar.

Os fluxos de $\mathrm{NH}_{3}$ calculados para a Baía de Guanabara variaram de 5 a $3486 \mu \mathrm{g} \mathrm{N} \mathrm{m}^{-2} \mathrm{~h}^{-1}$, atribuindo-se $c_{\text {ar }}=1 \mu \mathrm{g} \mathrm{m}^{-3}$, e de -20 a $3419 \mu \mathrm{g} \mathrm{N} \mathrm{m}^{-2} \mathrm{~h}^{-1}$, atribuindo-se $c_{\text {ar }}=5 \mu \mathrm{g} \mathrm{m}^{-3}$. O sinal negativo do fluxo denota transferência de $\mathrm{NH}_{3}^{\text {ar }}$ da atmosfera para o mar. As médias aritméticas dos fluxos calculados nas simulações foram, respectivamente, 482 e $447 \mu \mathrm{g} \mathrm{N} \mathrm{m}^{-2} \mathrm{~h}^{-1}$. Os fluxos foram calculados com base na média horária da velocidade do vento $(u)$ correspondente ao momento da coleta das amostras. Portanto, vale salientar que os valores calculados dos fluxos de $\mathrm{NH}_{3}$ de cada estação individualmente não são representativos das $24 \mathrm{~h}$ do dia em virtude das condicionantes meteorológicas, em especial a velocidade do vento. A direção do vento pode ter relação com a concentração de $\mathrm{NH}_{3}$ no ar, pois se espera que massas de ar de origem marinha possuam concentrações de $\mathrm{NH}_{3}$ inferiores às de origem continental. Durante as noites de inverno, a ocorrência de inversão térmica deve provocar aumento das concentrações de $\mathrm{NH}_{3}$ no ar.

Os fluxos de $\mathrm{NH}_{3}$ nas águas da Baía de Guanabara são muitas vezes superiores aos verificados em regiões oceânicas. Utilizando os modelos globais Moguntia/HAMOCC3, Quinn et al..$^{16}$ estimaram fluxos médios anuais de $\mathrm{NH}_{3}$ no Oceano Atlântico entre 0 e $1,5 \mathrm{mmol} \mathrm{m}^{-2}$ ano $^{-1}\left(0\right.$ e $\left.2,4 \mu \mathrm{g} \mathrm{N} \mathrm{m}^{-2} \mathrm{~h}^{-1}\right)$ para a faixa de latitude 20$60^{\circ} \mathrm{N}$, e entre $-1,5$ e $0 \mathrm{mmol} \mathrm{m}^{-2}$ ano $^{-1}\left(-2,4\right.$ e $\left.0 \mu \mathrm{g} \mathrm{N} \mathrm{m}^{-2} \mathrm{~h}^{-1}\right)$ para a faixa de latitude $5-20^{\circ} \mathrm{N}$. Durante ocorrências de florações fitoplanctônicas, Quinn et al. ${ }^{16}$ calcularam fluxos de $\mathrm{NH}_{3}$ até $12 \mu \mathrm{g}$ $\mathrm{N} \mathrm{m}^{-2} \mathrm{~h}^{-1}$, contudo, as concentrações de $\mathrm{NH}_{\mathrm{x}}$ na água do mar não atingiram valores superiores a $4 \mu \mathrm{mol} \mathrm{L} \mathrm{L}^{-1}$, valor 35 vezes inferior à concentração máxima de $\mathrm{NH}_{x}$ encontrada na Baía de Guanabara. No Mar do Norte, Asman et al. ${ }^{19}$ calcularam fluxos de $\mathrm{NH}_{3}$ que variaram de -44 e $25 \mu \mathrm{g} \mathrm{N} \mathrm{m}^{-2} \mathrm{~h}^{-1}$, onde as concentrações elevadas de $\mathrm{NH}_{3}$ no ar (de até $1,2 \mu \mathrm{g} \mathrm{NH} \mathrm{N}^{-3}$ ) em relação à concentração no ar em equilíbrio com a água do mar provocou um predomínio de transferência de $\mathrm{NH}_{3}$ da atmosfera para o mar, ou seja, um fluxo de $\mathrm{NH}_{3}$ de $-3,8 \mu \mathrm{g} \mathrm{N} \mathrm{m}^{-2} \mathrm{~h}^{-1}$. No Mar do Norte, as concentrações de $\mathrm{NH}_{3}$ no ar são geralmente elevadas devido à influência de massas de ar de origem continental e, por isso, os fluxos de $\mathrm{NH}_{3}$ se dão da atmosfera para o mar $^{19}$.

A Figura 4 mostra a sensibilidade teórica dos fluxos de $\mathrm{NH}_{3}$ à concentração de $\mathrm{NH}_{x}$ na água do mar e à velocidade do vento. A curva do fluxo $(F)$ em função da concentração de $\mathrm{NH}_{\mathrm{x}}$ foi calculada tendo-se fixado os valores de $T\left(28,0{ }^{\circ} \mathrm{C}\right), S(30,0), \mathrm{pH}(8,50), c_{\text {ar }}$ $\left(5,0 \mu \mathrm{g} \mathrm{NH}_{3} \mathrm{~m}^{3}\right)$ e $u\left(2,5 \mathrm{~m} \mathrm{~s}^{-1}\right)$, com a concentração de $\mathrm{NH}_{\mathrm{x}}$ variando de 0 a $160 \mu \mathrm{mol} \mathrm{L} \mathrm{L}^{-1}$. Para as condições estabelecidas, obteve-se $F=19,1\left[\mathrm{NH}_{\mathrm{x}}\right]_{\operatorname{mar}}-50,8$. Neste caso, aplicando-se a média aritmética calculada de $\mathrm{NH}_{\mathrm{x}}$ nas águas superficiais da baía (Tabela 1), obtém-se um fluxo de $\mathrm{NH}_{3}$ de $450 \mu \mathrm{g} \mathrm{m}^{-2} \mathrm{~h}^{-1}\left(370 \mu \mathrm{g} \mathrm{N} \mathrm{m}^{-2} \mathrm{~h}^{-1}\right)$. Da mesma forma, a curva de $F$ em função da velocidade do vento $(u)$ foi calculada para os mesmos valores de $T, S, \mathrm{pH}$ e $c_{\mathrm{ar}}$, fixando-se
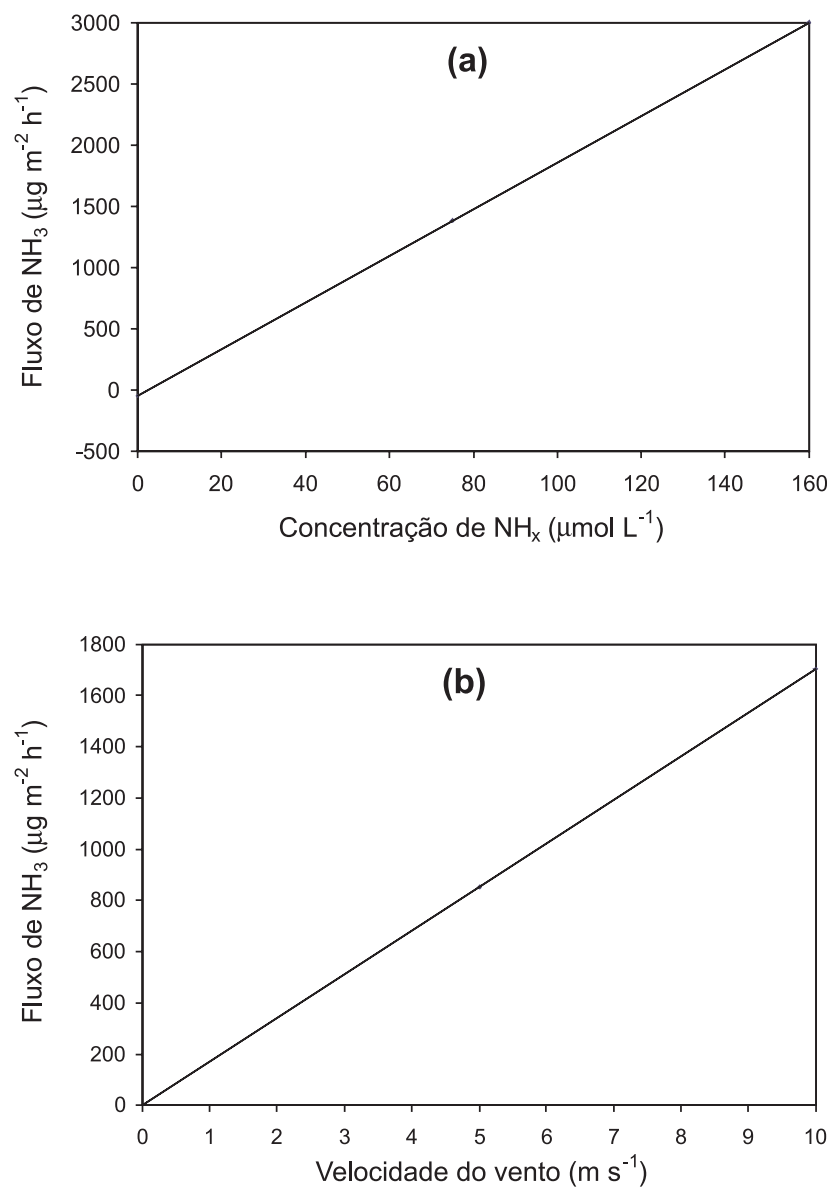

Figura 4. Sensibilidade do fluxo de $\mathrm{NH}_{3}$ na interface ar-mar em relação à concentração de nitrogênio amoniacal $\left(\mathrm{NH}_{x}\right)$ na água do mar (a) e à velocidade do vento (b). Para a curva do fluxo de $\mathrm{NH}_{3}$ em função da concentração de $N_{x}: T=28,0^{\circ} \mathrm{C} ; \mathrm{S}=30,0 ; \mathrm{pH}=8,50 ; c_{a r}=5,0 \mu \mathrm{g} \mathrm{NH} \mathrm{H}_{3} \mathrm{~m}^{-3}$ e u $=2,5 \mathrm{~m} \mathrm{~s}^{-1}$. Para a curva do fluxo de $\mathrm{NH}_{3}$ em função da velocidade do vento: $\mathrm{T}=28,0^{\circ} \mathrm{C}$; $S=30,0 ; p H=8,50 ; c_{a r}=5,0 \mu g \mathrm{NH}_{3} m^{-3} e\left[\mathrm{NH}_{x}\right]=25 \mu \mathrm{mol} \mathrm{L} L^{-1}$ 
Tabela 2. Fluxos $(F)$ de $\mathrm{NH}_{3}$ na interface ar-mar nas 37 estações de amostragem na Baía de Guanabara no período de 5 a 7 de abril de 2004

\begin{tabular}{|c|c|c|c|c|c|c|c|}
\hline Data & Estação & Direção do vento & $\begin{array}{c}c_{\text {eq }} \\
\mu \mathrm{g} \mathrm{m}^{-3}\end{array}$ & $\begin{array}{c}u \\
\mathrm{~m} \mathrm{~s}^{-1}\end{array}$ & $\begin{array}{c}k_{\mathrm{a}} \\
\mathrm{m} \mathrm{s}^{-1}\end{array}$ & 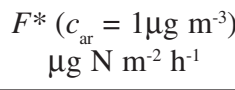 & 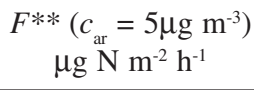 \\
\hline 07/04/04 & 1 & W & 7,4 & 1,9 & 0,16 & 41 & 15 \\
\hline 07/04/04 & 2 & W & 11,8 & 1,9 & 0,16 & 68 & 43 \\
\hline 07/04/04 & 3 & W & 9,0 & 2,5 & 0,22 & 67 & 34 \\
\hline 07/04/04 & 4 & NW & 3,1 & 1,8 & 0,16 & 12 & -12 \\
\hline 07/04/04 & 5 & NW & 9,0 & 1,8 & 0,16 & 48 & 24 \\
\hline 07/04/04 & 6 & NW & 1,8 & 1,9 & 0,16 & 5 & -20 \\
\hline 07/04/04 & 7 & NW & 2,6 & 2,1 & 0,18 & 11 & -17 \\
\hline 05/04/04 & 8 & SE & 9,3 & 2,1 & 0,18 & 58 & 30 \\
\hline 07/04/04 & $8 \mathrm{c}$ & NW & 9,7 & 1,8 & 0,16 & 52 & 28 \\
\hline 05/04/04 & 9 & SE & 10,1 & 2,1 & 0,18 & 65 & 36 \\
\hline 05/04/04 & 10 & SE & 290,2 & 2,1 & 0,18 & 2049 & 2021 \\
\hline 07/04/04 & $10 \mathrm{c}$ & SW & 7,8 & 3,0 & 0,26 & 68 & 28 \\
\hline 07/04/04 & 11 & SW & 39,4 & 2,5 & 0,22 & 321 & 288 \\
\hline 05/04/04 & 12 & $\mathrm{E}$ & 15,8 & 1,5 & 0,13 & 75 & 55 \\
\hline 05/04/04 & 13 & SE & 12,0 & 1,9 & 0,17 & 71 & 45 \\
\hline $05 / 04 / 04$ & 14 & SE & 8,9 & 1,9 & 0,17 & 51 & 25 \\
\hline $05 / 04 / 04$ & 15 & SE & 15,2 & 1,9 & 0,17 & 92 & 66 \\
\hline $05 / 04 / 04$ & 16 & $\mathrm{E}$ & 15,7 & 1,5 & 0,13 & 75 & 54 \\
\hline $05 / 04 / 04$ & 17 & SE & 25,5 & 2,7 & 0,23 & 221 & 185 \\
\hline 05/04/04 & $17 \mathrm{~A}$ & SE & 128,6 & 2,7 & 0,23 & 1152 & 1116 \\
\hline 05/04/04 & 18 & SE & 147,7 & 2,7 & 0,23 & 1324 & 1288 \\
\hline $05 / 04 / 04$ & 19 & $\mathrm{E}$ & 166,5 & 2,1 & 0,18 & 1134 & 1107 \\
\hline $05 / 04 / 04$ & 20 & $\mathrm{E}$ & 132,1 & 2,1 & 0,18 & 899 & 871 \\
\hline 06/04/04 & 23 & $\mathrm{E}$ & 34,4 & 1,3 & 0,11 & 141 & 124 \\
\hline 06/04/04 & 24 & NE & 34,3 & 1,9 & 0,16 & 209 & 184 \\
\hline 05/04/04 & 25 & SE & 210,4 & 5,0 & 0,43 & 3486 & 3419 \\
\hline 06/04/04 & 26 & SE & 13,6 & 3,2 & 0,28 & 134 & 91 \\
\hline 06/04/04 & 27 & SE & 11,7 & 3,2 & 0,28 & 114 & 71 \\
\hline 06/04/04 & 28 & $\mathrm{NE}$ & 13,4 & 1,9 & 0,16 & 78 & 53 \\
\hline 06/04/04 & 29 & $\mathrm{~N}$ & 44,9 & 5,0 & 0,43 & 731 & 665 \\
\hline 06/04/04 & 30 & SE & 162,1 & 4,4 & 0,38 & 2370 & 2311 \\
\hline 06/04/04 & 33 & SE & 11,4 & 3,2 & 0,28 & 110 & 68 \\
\hline 06/04/04 & 34 & SE & 12,3 & 4,2 & 0,36 & 157 & 102 \\
\hline 06/04/04 & 35 & SE & 16,7 & 4,2 & 0,36 & 219 & 163 \\
\hline 06/04/04 & 36 & SE & 48,3 & 4,4 & 0,38 & 702 & 644 \\
\hline 06/04/04 & 37 & SE & 85,3 & 4,4 & 0,38 & 1239 & 1180 \\
\hline 06/04/04 & 42 & SE & 14,3 & 4,4 & 0,38 & 196 & 137 \\
\hline \multicolumn{3}{|c|}{ Média aritimética } & 48,5 & 2,7 & 0,2 & 482 & 447 \\
\hline \multicolumn{3}{|l|}{$\mathrm{DP}$} & 68,8 & 1,1 & 0,1 & 773 & 766 \\
\hline \multicolumn{3}{|l|}{ Mediana } & 14,3 & 2,1 & 0,2 & 114 & 71 \\
\hline \multicolumn{3}{|l|}{ Mínimo } & 1,8 & 1,3 & 0,1 & 5 & -20 \\
\hline \multicolumn{3}{|l|}{ Máximo } & 290,2 & 5,0 & 0,4 & 3486 & 3419 \\
\hline
\end{tabular}

$c_{\text {eq }}=$ concentrações teóricas de $\mathrm{NH}_{3}$ no ar na condição de equilíbrio com as concentrações medidas de $\mathrm{NH}_{\mathrm{x}}$ nas águas superficiais; $u=$ velocidade do vento; $k_{\mathrm{a}}$ velocidade de transferência; $F^{*}$ e $F^{* *}=$ fluxos cálculados adotando-se concentrações atmosféricas de $\mathrm{NH}_{3}$ de 1 e $5 \mu \mathrm{g} \mathrm{m}^{-3}$, respectivamente.

também [NH $]\left(25 \mu \mathrm{mol} \mathrm{L} \mathrm{L}^{-1}\right)$, com $u$ variando de 0 a $10 \mathrm{~m} \mathrm{~s}^{-1}$. Para as condições estabelecidas, obteve-se $F=170,4 u$. Neste caso, considerando-se que as velocidades médias dos ventos nas proximidades da entrada da Baía de Guanabara durante o período de verão e outono podem variar de 2,8 a $4,7 \mathrm{~m} \mathrm{~s}^{-1} 48$, calcula-se, para este período do ano, valores de fluxo de $\mathrm{NH}_{3}$ entre 477 e $801 \mu \mathrm{g}$ $\mathrm{m}^{-2} \mathrm{~h}^{-1}$ (393 e $660 \mu \mathrm{g} \mathrm{N} \mathrm{m}^{-2} \mathrm{~h}^{-1}$ ). Como foi demonstrado, o fluxo de $\mathrm{NH}_{3}$ cresce linearmente em função das duas variáveis, $\mathrm{NH}_{\mathrm{x}} \mathrm{e} u$, e, teoricamente, o fluxo de $\mathrm{NH}_{3}$ é mais sensível às variações da velocidade do vento (Figura 4).

Para uma avaliação mais precisa dos fluxos de $\mathrm{NH}_{3}$ nas águas da Baía de Guanabara e extrapolação desses valores para estimativa da taxa de emissão na escala de tempo anual faz-se necessário medi- das, em escala temporal, das concentrações de $\mathrm{NH}_{x}$ nas águas superficiais da baía; medidas, em escala temporal, das concentrações de $\mathrm{NH}_{3}$ na atmosfera superficial e a relação de seus valores com as direções dos ventos predominantes e, desenvolvimento de uma técnica capaz de medir diretamente o fluxo de $\mathrm{NH}_{3}$ na interface ar-mar de forma a fornecer o valor do fluxo, especialmente nas áreas mais poluídas, na condição de $u=0$, ou seja, calmaria absoluta.

\section{Tentativa preliminar de balanço parcial de nitrogênio}

Considerando-se os valores médios do fluxo de $\mathrm{NH}_{3}$ na interface ar-mar na Baía de Guanabara de 450 e $480 \mu \mathrm{g} \mathrm{m}^{-2} \mathrm{~h}^{-1}$ e a área de espelho d'água da baía de $328 \mathrm{~km}^{2}$, estima-se uma taxa de emissão 
de 3,5 a 3,8 $t \mathrm{~N} \mathrm{dia}^{-1}$, o que representa de 2,5 a 2,9\% da descarga de $\mathrm{N}$ total lançada na Baía de Guanabara através dos rios que, de acordo com as estimativas da $\mathrm{JICA}^{25}$, é da ordem de 130-140 $t \mathrm{~N} \mathrm{dia}^{-1}$.

Uma taxa de emissão de $\mathrm{NH}_{3}$ da ordem de 3,5 $t \mathrm{~N}$ dia $^{-1}$ é 5 vezes maior que a taxa de deposição atmosférica de $\mathrm{N}$ inorgânico $\left(\mathrm{NH}_{4}^{+}+\mathrm{NO}_{3}^{-}\right)$transferido, através da chuva (deposição úmida), da atmosfera diretamente para as águas da Baía de Guanabara. A taxa de deposição úmida de $\mathrm{N}$ inorgânico é em torno de $0,7 t \mathrm{~N}_{\text {dia }}{ }^{-1}$ valor calculado com base na área de espelho d'água da baía (328 $\mathrm{km}^{2}$ ) e um fluxo atmosférico, via deposição úmida somente, de 7,3 $\mathrm{kg} \mathrm{N} \mathrm{ha}^{-1}$ ano $^{-1}\left(\sim 70 \% \mathrm{NH}_{4}^{+} \mathrm{e} \sim 30 \% \mathrm{NO}_{3}^{-}\right)^{49}$.

No caso de uma tentativa de obtenção da taxa anual de emissão de $\mathrm{NH}_{3}$ da baía de Guanabara com base nos resultados obtidos neste trabalho, deve-se considerar que durante o período de inverno as concentrações de $\mathrm{NH}_{\mathrm{x}}$ nas águas superficiais da baía podem ser mais altas que as verificadas neste trabalho no início do mês de abril, condição que teoricamente implica em um aumento dos fluxos de $\mathrm{NH}_{3}$, conforme simulação demonstrada pela Figura 4a.

\section{CONCLUSÕES}

As concentrações de $\mathrm{N}$ amoniacal $\left(\mathrm{NH}_{\mathrm{x}}\right.$ ) nas águas superficiais da Baía de Guanabara apresentam uma grande variação espacial, com os maiores valores verificados nos arredores da Ilha do Governador, especialmente no trecho entre a Ilha do Governador e o continente. Os fluxos de $\mathrm{NH}_{3}$ na interface ar-mar mostraram ter como principais fatores de controle, no caso da Baía de Guanabara, a concentração de $\mathrm{NH}_{x}$ nas águas superficiais, que variou de 2 a quase $150 \mu \mathrm{mol} \mathrm{L}{ }^{-1}$, e a velocidade do vento. Com simulações efetudas para concentrações atmosféricas (não medidas) de $\mathrm{NH}_{3}$ de 1 e $5 \mu \mathrm{g} \mathrm{m}^{-3}$, os fluxos médios calculados variaram de 450 a 480 $\mu \mathrm{g} \mathrm{N} \mathrm{m}{ }^{-2} \mathrm{~h}^{-1}$, correspondendo a uma taxa de emissão de aproximadamente 3,5 a 3,8 $t \mathrm{~N} \mathrm{dia}^{-1}$, valor superior ao que as águas da Baía de Guanabara recebem, em média, de $\mathrm{N}$ inorgânico $\left(\mathrm{NH}_{4}^{+} \mathrm{e} \mathrm{NO}_{3}^{-}\right)$ diretamente através das chuvas.

\section{AGRADECIMENTOS}

Ao Prof. E. D. Barbiére, do Departamento de Geoquímica da UFF, e às Profas. C. M. Paiva e A. M. P. de Ávila, do Departamento de Meteorologia da UFRJ, pela concessão dos dados meteorológicos, e ao $\mathrm{CNPq}$ pelo apoio financeiro concedido através dos Projetos 474113/2003-5 e 521907/96-9.

\section{REFERÊNCIAS}

1. Finlayson-Pitts, B. J.; Pitts Jr., J. N.; Chemistry of the upper and lower atmosphere, Academic Press: San Diego, 2000.

2. Lee, D. S.; Halliwell, C.; Garland, J. A.; Dollard, G. J.; Kingdon, R. D.; Atmos. Environ. 1998, 32, 431.

3. Oliver, J. G. J.; Bouwman, A. F.; van der Hoek, K. W.; Berdowski, J. J. M.; Environ. Pollut. 1998, 102, 135.

4. Krupa, S. V.; Environ. Pollut. 2003, 124, 179.

5. Felix, E. P.; Cardoso, A. A.; Quim. Nova 2004, 27, 123.

6. Figuerêdo, D. V.; Ambio 1999, 28, 514.

7. de Mello, W. Z.; Environ. Pollut. 2001 114, 235.

8. Lara, L. B. L. S.; Artaxo, P.; Martinelli, L. A.; Victoria, R. L.; Camargo, P. B.; Krusche, A.; Ayers, G. P.; Ferraz, E. S. B.; Ballester, M. V.; Atmos. Environ. 2001, 35, 4937.

9. Fornaro, A.; Gutz, I. G. R.; Atmos. Environ. 2003, 37, 117.

10. Rocha, F. R.; Fracassi da Silva, J. A.; Lago, C. L.; Fornaro, A.; Gutz, I. G. R.; Atmos. Environ. 2003, 37, 105.

11. Migliavacca, D.; Teixeira, E. C.; Pires, M.; Fachel, J.; Atmos. Environ. 2004, 38,1641 .

12. Schuurkes, J. A. A. R.; Mosello, R.; Schweiz. Z. Hydrol. 1988, 50, 71.
13. Matson, P. A.; McDowell, W. H.; Townsend, A. R.; Vitousek, P. M.; Biogeochemistry 1999, 46, 67.

14. Stumm, W.; Morgan, J. J.; Aquatic Chemistry - An Introduction Emphasizing Chemical Equilibria in Natural Waters, John Wiley \& Sons: New York, 1981.

15. Baumgarten, M. D. G. Z.; Rocha, J. M. D. B.; Niencheski, L. F. H.; Manual de Análises em Oceanografia Química, Ed. FURG: Rio Grande, 1996.

16. Quinn, P. K.; Barret, K. J.; Dentener, F. J.; Lipschultz, F.; Six, K. D.; Biogeochemistry 1996, 35, 275.

17. Liss, P. S.; Slater, P. G.; Nature 1974, 247, 181.

18. Duce, R. A.; Liss, P. S.; Merrill, J. T.; Atlas, E. L.; Buat-Menard, P.; Hicks, B. B.; Miller, J. M.; Prospero, J. M.; Arimoto, R.; Church, T. M.; Ellis, W.; Galloway, J. N.; Hansen, L.; Jickells, T. D.; Knap, A. H.; Reinhardt, K. H.; Schneider, B.; Soudine, A.; Tokos, J. J.; Tsunogai, S.; Wollast, R.; Zhou, M.; Global Biogeochem. Cycles 1991, 5, 193.

19. Asman, W. A. H.; Harrison, R. M.; Ottley, C. J.; Atmos. Environ. 1994, 28,3647 .

20. FEEMA - Fundação Estadual de Engenharia do Meio Ambiente; Projeto de Recuperação Gradual do Ecossistema da Baía de Guanabara Indicadores Ambientais de Degradação de Obras e Projetos de Recuperação, Rio de Janeiro, 1990.

21. Mayr, L. M.; Tenenbaum, R. D.; Villac, M. C.; Paranhos, R.; Nogueira, C. R.;. Bonecker, S. L. C.; Bonecker, A.C.T. Em Coastilines of Brazil; Neves, C.; Magoon, O. T., eds.; American Society of Civil Engineers: New York, 1989.

22. Lavrado, H. P.; Mayr, L. M.; Carvalho, V.; Paranhos, R.; Proceedings of the Seventh Symposium on Coastal and Ocean Management, Long Beach, California, 1991.

23. Paranhos, R.; Mayr, L. M.; Fresenius Environ. Bull. 1993, 2, 647.

24. Paranhos, R.; Mayr, L. M.; Lavrado, H. P.; Castilho, P. C.; Arq. Biol. Tecnol. 1993, 36, 685 .

25. JICA - Japan International Cooperation Agency; The Study on Recuperation of the Guanabara Bay Ecossystem, Kokusai Kogyo, 1994.

26. Kjerfve, B.; Ribeiro, C. H. A.; Dias, G. T. M.; Filipo, A. M.; Quaresma, V. S.; Cont. Shelf Res. 1997, 17, 1609.

27. FEEMA - Fundação Estadual de Engenharia do Meio Ambiente; Qualidade das Águas da Baía de Guanabara, Rio de Janeiro, 2000.

28. Paranhos, R.; Pereira, A. P.; Mayr, L. M.; Environ. Monit. Assess. 1998, $50,131$.

29. Valentin, J.; Tenenbaum, D.; Bonecker, A.; Bonecker, S.; Nogueira, C.; Paranhos, R.; Villac, M. -C.; J. Rech. Océanographique 1999, 24, 33.

30. Campos, V. P.; Tese de Doutorado, Universidade Federal da Bahia, Brasil, 1995.

31. Costa, A. M.; Dissertação de Mestrado, Universidade Federal da Bahia, Brasil, 2002.

32. Fornaro, A.; Souza, S. R.; Vasconcellos, P. C.; Bruni, H. S.; Montero, L.; Carvalho, L. R. F.; Gutz, I. G. R.; Andrade, M. F.; Gonçalves, F. L. T.; Resumos da $23^{a}$ Reunião Anual da Sociedade Brasileira de Química, Poços de Caldas, Brasil, 2000.

33. Maia, L. F. P. G.; comunicação pessoal.

34. CIDS - Centro Internacional de Desenvolvimento Sustentável; Baía de Guanabara - Dossiê Sócio-Ambiental, Rio de Janeiro, 2000.

35. http://www.dhn.mar.mil.br/chm/tabuas/, acessada em Abril 2004.

36. FAO - Food and Agriculture Organization; FAO Fish. Tech. Pap. 1975, 137, 238.

37. Dasgupta, P. K.; Dong, S.; Atmos. Environ. 1986, 20, 565

38. Randal, M.; Failey, C. F.; Chem. Rev. 1927, 4, 271.

39. Garrels, R. M.; Christ, C. L.; Solutions, Minerals, and Equilibria, Freeman, Cooper \& Company: San Francisco, 1965.

40. Lyman, J.; Fleming, R. H.; J. Marine Res. 1940, 3, 134.

41. Manov, G. G.; Bates, R. G.; Hamer, W. J.; Acree, S. F.; J. Am. Chem. Soc. 1943, 65, 1765.

42. Bates, R. G.; Pinching, G. D.; Am. Chem. J. 1950, 72, 1393.

43. Mayr, L. M.; Tese de Doutorado, Universidade Federal do Rio de Janeiro, Brasil, 1998.

44. Quiroga, A. I. P.; Dissertação de Mestrado, Universidade Santa Úrsula, Brasil, 1999.

45. Contador, L. S.; Dissertação de Mestrado, Universidade Santa Úrsula, Brasil, 2000.

46. Sorensen, L. L.; Hertel, O.; Skjoth, C. A.; Lund, M.; Pedersen, B.; Atmos. Environ. 2003, 37, 167.

47. Santos, E. S.; Dissertação de Mestrado, Universidade Federal Fluminense, Brasil, 2001.

48. Barbiére, E. B.; Kronemberger, D. M. P.; Cad. Geoc. 1994, 12, 57.

49. de Mello, W. Z.; Resumos do IX Congresso Brasileiro de Geoquímica, Belém, Brasil, 2003. 